\title{
Magnetic Resonance (MR) Imaging of the cardiac venous system and MR-guided intubation of the coronary sinus in swine: a feasibility-study
}

\author{
Mirja Neizel*1, Nils Kraemer ${ }^{2}$, Jan Balzer ${ }^{1}$, Bernhard Schnackenburg3, \\ Malte Kelm¹, Rolf W Guenther ${ }^{2}$, Harald P Kuehl ${ }^{2}$ and Gabriele A Krombach ${ }^{2}$
}

Address: ${ }^{1}$ University Hospital Duesseldorf, Duesseldorf, Germany, ${ }^{2}$ University Hospital Aachen, Duesseldorf, Germany and ${ }^{3}$ Philips Medical Systems, Hamburg, Germany

* Corresponding author

from I3th Annual SCMR Scientific Sessions

Phoenix, AZ, USA. 21-24 January 2010

Published: 21 January 2010

Journal of Cardiovascular Magnetic Resonance 2010, I2(Suppl I):O23 doi:10.1 I86/I532-429X-I2-SI-O23

This abstract is available from: http://jcmr-online.com/content//2/SI/O23

(c) 2010 Neizel et al; licensee BioMed Central Ltd.

\section{Introduction}

Procedures like cardiac resynchronization therapy (CRT) or percutanous transcatheter mitral annuloplasty where improved 3-dimensional knowledge about cardiac and cardiac vein anatomy is needed may benefit from magnetic resonance (MR)-guidance.

\section{Purpose}

The aim of this study was to visualize the coronary sinus using MR imaging and to demonstrate the feasibility of MR-guided intubation of the cardiac venous system (CVS) in swine.

\section{Methods}

6 pigs were investigated. All experiments were performed using an interventional 1.5 Tesla MRI system. The CVS was visualized using an inversion-recovery navigatorgated whole-heart steady-state free precession sequence after administration of Gd-BOPTA contrast agent (130150 slices, voxel size $1.2 \times 1.2 \times 1.8 \mathrm{~mm}^{3}$, TR/TE 6.4/3.2, flip angle $85^{\circ}$. Parallel imaging was used with a SENSEfactor of 2 . The coronary sinus was then intubated under MR-guidance with a passive MR-compatible guidewire modified by incorporation of iron oxide markers for improved visualization and a non-braded Cobra-catheter. MR-guided interventions were monitored using a steadystate free precession real-time imaging sequence. Parameters of the real-time imaging sequence were $\mathrm{TR} / \mathrm{TE}=2.5 /$
$1.25 \mathrm{~ms}$, flip angle $45^{\circ}$, slice thickness $8 \mathrm{~mm}$, matrix 128 $\times 128$ reconstructed to $256 \times 256$ using zero-filling. Planning of the image planes, especially for the coronary sinus, were performed with the help of the wholeheart acquisition. Time needed was measured for MR-guided intubation of the CVS and compared to the time needed for fluoroscopy guided intubation of the CVS.

\section{Results}

The CVS could be visualized in all animals (100\%). The diameter of the ostium of the coronary sinus was $7.6 \pm$ $0.75 \mathrm{~mm}$ measured using the inversion-recovery 3-dimensional steady-state free-precession balanced wholeheart sequence. Intubation of the coronary sinus and its site branches was also feasible in all cases. Time spent for MRguided intubation of the CVS was comparable to time spent for fluoroscopy-guided intubation $(8.2 \pm 2$ min versus $8.3 \pm 1.3$ min; $\mathrm{p}=0.92$ ).

\section{Conclusion}

MR-visualization and MR-guided intubation of the coronary sinus and its side branches is feasible. The feasibility of MR-guided intubation of the CVS might have some relevance for procedures like CRT, requiring improved 3dimensional knowledge about cardiac and cardiac vein anatomy as well as information about function, myocardial scar and asynchrony in the near future. 